\title{
The MoEDAL experiment at the LHC
}

\section{Searching beyond the standard model}

\author{
James L. Pinfold (for the MoEDAL Collaboration) ${ }^{1, a}$ \\ ${ }^{1}$ University of Alberta, Physics Department, Edmonton, Alberta T6G OV1, Canada
}

\begin{abstract}
MoEDAL is a pioneering experiment designed to search for highly ionizing avatars of new physics such as magnetic monopoles or massive (pseudo-)stable charged particles. Its groundbreaking physics program defines a number of scenarios that yield potentially revolutionary insights into such foundational questions as: are there extra dimensions or new symmetries; what is the mechanism for the generation of mass; does magnetic charge exist; what is the nature of dark matter; and, how did the big-bang develop. MoEDAL's purpose is to meet such far-reaching challenges at the frontier of the field.

The innovative MoEDAL detector employs unconventional methodologies tuned to the prospect of discovery physics. The largely passive MoEDAL detector, deployed at Point 8 on the LHC ring, has a dual nature. First, it acts like a giant camera, comprised of nuclear track detectors - analyzed offline by ultra fast scanning microscopes - sensitive only to new physics. Second, it is uniquely able to trap the particle messengers of physics beyond the Standard Model for further study. MoEDAL's radiation environment is monitored by a state-of-the-art real-time TimePix pixel detector array. A new MoEDAL sub-detector to extend MoEDAL's reach to millicharged, minimally ionizing, particles (MMIPs) is under study Finally we shall describe the next step for MoEDAL called Cosmic MoEDAL, where we define a very large high altitude array to take the search for highly ionizing avatars of new physics to higher masses that are available from the cosmos.
\end{abstract}

\section{Introduction}

In 2010 the MoEDAL experiment [1] at the Large Hadron Collider (LHC) was unanimously approved by CERN's Research Board to start data taking in 2015. MoEDAL is a pioneering experiment designed to search for highly ionizing avatars of new physics such as magnetic monopoles or massive (pseudo-)stable charged particles. Its groundbreaking physics program defines over 30 scenarios that yield potentially revolutionary insights into such foundational questions as: are there extra dimensions or new symmetries; what is the mechanism for the generation of mass; does magnetic charge exist; what is the nature of dark matter; and, how did the big-bang develop. The LHC machine [2] brings its counter rotating beams nominally consisting of 2808 bunches of protons each with roughly $10^{11}$ protons per bunch. The proton beams are brought into collision at four points around the $27 \mathrm{~km}$ circumference LHC ring. The biggest of the LHC's seven experiments are ATLAS [3] and CMS [4], general-purpose detectors designed to investigate the largest range of physics possible. ALICE [5]

a e-mail: jpinfold@ualberta.ca 
and $\mathrm{LHCb}$ [6] have detectors specialized for focussing on specific phenomena such as quark-gluon plasma formation and the investigation of the matter-antimatter asymmetry using b-hadron physics, respectively. These four detectors in huge caverns sit roughly $100 \mathrm{~m}$ underground on the LHC ring. Prior to the approval of the MoEDAL experiment the smallest experiments on the LHC were TOTEM [7] and LHCf [8], which focus on "forward particles" - protons or heavy ions that brush past each other rather than meeting head on when the beams collide. TOTEM uses detectors positioned on either side of the CMS interaction point, while LHCf is made up of two detectors that sit along the LHC beam line, at 140 metres either side of the ATLAS collision point. MoEDAL the seventh and latest LHC experiment shares an interaction point with the LHCb experiment. It is designed to significantly expand the discovery horizon of the general-purpose LHC detectors, ATLAS and CMS in a complementary manner. The innovative MoEDAL detector employs unconventional methodologies tuned to the prospect of discovery physics. The largely passive MoEDAL detector, deployed at Point 8 on the LHC ring has a dual nature. First, it acts like a giant camera, comprised of Nuclear Track Detectors (NTDs) - analyzed offline by ultra fast scanning microscopes - sensitive only to new physics. Second, it is uniquely able to trap the particle messengers of physics beyond the Standard Model for further study. MoEDAL's radiation environment is monitored by a state-of-the-art real-time TimePix pixel detector array. In addition, we are preparing a proposal for a third active detector element capable of detecting the faintest flicker of light - single photon signals - produced by the passage of Minimally Ionizing (millicharged) Particles (mQPs) with charge as small as one thousandth that of an electron.

\section{Highly ionzing particles - messengers of new physics}

MoEDAL's groundbreaking physics prospectus [9] covers 34 fundamentally important Beyond the Standard Model (BSM) scenarios involving electrically and magnetically charged particles. It is designed to exploit the use of Highly Ionizing Particles (HIPs) as wide ranging avatars of new physics. One of the main objectives for MoEDAL is the state-of-the-art search for the magnetic charge carried by the monopole, just as the search for the Higgs was the prime motivation for the LHC and its general-purpose experiments.

To be detected a particle must interact with the material of the detector losing energy as they travel. Except when they are moving very near to the speed of light charged particles heavier than electrons primarily lose energy by interacting with the atoms of the material by exciting atomic electrons or ionizing atoms. The Bethe formula describes the energy loss per distance travelled of fast charged particles that are more massive than electrons such as muons, protons, pions and ions (atomic nuclei). A simplified form of the Bethe equation is:

$$
-\frac{d E}{d x}=z^{2} \frac{K Z}{A \beta^{2}}\left[\frac{1}{2} \ln \left(\frac{2 m_{e} \beta^{2} \gamma^{2}}{I^{2}}\right)-\beta^{2}\right]
$$

where, $\beta$ is the speed of the particles expressed as a fraction of the speed of light $(\beta=\mathrm{v} / \mathrm{c})$ and $K=$ $4 \pi N_{A} r_{e}^{2} m_{e} c^{2}$. The properties of the medium are the atomic number $Z$, the atomic mass $A$ and the mean excitation energy $I$. The constants are the electron mass, $m_{e}$, the classical electron radius, $r_{e}$ and Avogardo's number $N_{A}$.

All stable or long-lived Standard Model charged particles produced in proton-proton collisions at the LHC have a single charge $(z e \rightarrow e)$, are relatively light i.e. at most the mass of a proton and are moving near to the speed of light $(\beta \sim 1$ ). Particle are "minimum ionizing" (MIPs) if $\beta \gamma=3 \rightarrow 4$ (or $d E / d x \sim 1 \rightarrow 2 \mathrm{MeV} \mathrm{g}^{-1} \mathrm{~cm}^{2}$ ).

In proton-proton collisions as the LHC electrically charged particles can only be highly ionizing if they are massive and thus moving very slowly with $\beta \gamma<1$, and/or multiply charged. However, there 
are no known massive stable or long-lived particles that are multiply charged. The only way that a Standard Model particle can be highly ionizing is if it is slowing down to stop. Energy loss due to ionization by $\mathrm{mQP}$ is described by the Bethe-Bloch formula. Thus, all other things being equal a milli-charged particle ( with $\mathrm{q}=0.001 \mathrm{e}$ ) will ionize $10^{-6}$ aless than a singly charged particle (with $\mathrm{q}=1 \mathrm{e}$ ) and multiple detection coincidence can rule out detector related backgrounds. Thus, massive stable or pseudo-stable HIPs or mQps traversing the detector are clear messengers of new physics with no obvious Standard Model Backgrounds.

\subsection{Particles with magnetic charge}

A prime example of a HIP from BSM is the magnetic monopole. The ionization energy loss of a magnetically charged particle can be obtained from the Bethe formula by replacing the electric charge $z e$ by $n g \beta$ where $g$ is the magnetic charge and $\mathrm{n}$ is the number of Dirac magnetic charges and realizing that for magnetic monopoles the velocity dependence of the Lorentz force cancels the $1 / \beta^{2}$ term in the Bethe-Bloch formula for charged particles:

$$
-\frac{d E}{d x}=n(g / e)^{2} \frac{K Z}{A}\left[\frac{1}{2} \ln \left(\frac{2 m_{e} \beta^{2} \gamma^{2}}{I^{2}}\right)-\beta^{2}\right]
$$

A single Dirac (magnetic) charge is $g \approx 68.5 e$. As we can see from the Bethe formula for magnetic charge given above, the energy loss of a singly charged $(n=1)$ very relativistic magnetic monopole $(\beta \approx 1)$ is amazingly about 4700 times that of a proton.

The MoEDAL experiment detects HIPs using stacks of passive plastic Nuclear Track Detectors (NTDs). The HIP damages the plastic as it traverses the NTD stack. Etching the plastic in a hot sodium hydroxide solution can reveal this damage as two collinear etch-pits each with diameter $\sim 5$ $10 \mu \mathrm{m}$ in each of the six layers of the stack. The NTD plastic employed by MoEDAL has a threshold of around $Z / \beta$ of 5 (or 5 MIPs) where $Z$ is the charge number of the particle and $\beta$ is the velocity of the particle divided by the speed of light $(v / c)$. A HIP messenger of new physics would leave a tell tale signature of twelve tiny etch pits (for each stack of ten NTD sheets) forming a precisely defined line pointing back to the collision. There is no known Standard Model particle that can give a trail of collinear etch pits in a MoEDAL stack.

\section{Directly detecting magnetic charge}

Clearly, a unique property of the magnetic monopole is that it has magnetic charge. Thus a moving magnetic monopole will be ringed by an electric field just as a moving electric charge is ringed by a magnetic field. Imagine that a magnetic monopole traverses the superconducting wire coil of a SQUID. As the monopole approaches the coil it drives an electrical current within the coil that in turn creates a changing magnetic field. This changing magnetic field creates an electric field that counters the original electric field of the moving monopole. As the wire in the coil has no resistance the overall effect is to make the total electric field vanish in the coil. Meanwhile the current continues to flow in the coil because the wire is superconducting and without resistance. One can use Faraday's Law to calculate the magnitude of the current that would be generated:

$$
I=-\frac{m u_{0} q_{m}}{L}
$$

where, $L$ is the inductance of the coil, $\mu_{0}$ is the permeability of free space and $q_{m}$ is the magnetic charge. Consequently, the current induced only depends on the magnetic charge and is independent of the speed, mass or direction of the magnetic monopole. 


\section{A description of the MoEDAL detector}

The novel MoEDAL detector is deployed at IP8 on the LHC ring, depicted in figure 1, is comprised of three key subsystems. The main component of MoEDAL's NTD subsystem consists of a low threshold (LT) NTD array - comprised of an array of (400) plastic NTD stacks $\left(25 \times x 25 \mathrm{~cm}^{2}\right)$ each stack made up of CR39 (3) and MAKROFOL (3) NTD sheets. This is the largest NTD array every deployed at an accelerator. The CR39 NTDs have an excellent charge resolution ( $\geq 0.2$ of a single electric charge, based on a single measurement) and a low threshold, allowing the detection of HIPs with and ionizing power equal to or more than 5 times that of a Minimum Ionizing Particle (MIP). An illustration of the LT-NTD stack is given in figure 2 (Left).

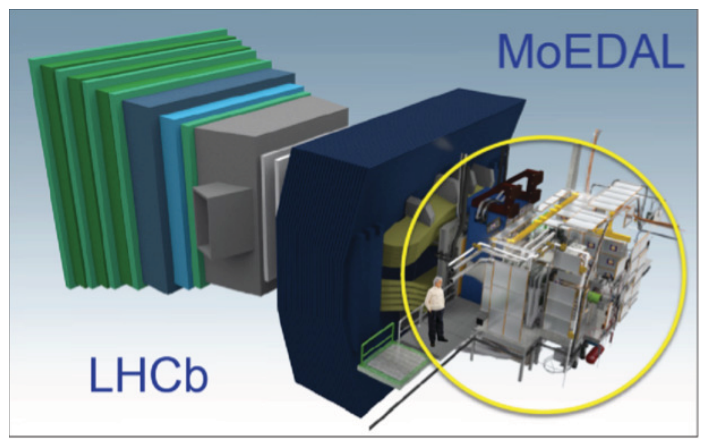

Figure 1. A view of the MoEDAL detector deployed around the LHCb's VELO detector at IP8.

The LT-NTD array has been enhanced by the Very High Charge Catcher (VHCC) subdetector with threshold around 50 MIPs. The VHCC subdetector - comprised of two flexible low mass stacks of MAKROFOL in an aluminium foil envelope - is deployed in the forward acceptance of the LHCb experiment just after the LHCb RICH detector. The overall acceptance of the NTD system is $~ 60 \%$. The exposed NTD detectors will be etched to reveal the passage of a HIP as illustrated in figure 2 (right). The etched plastic will then be analysed at Bologna and CERN using state-of-the art ultra-fast automated optical scanning microscopes developed by the Muenster and INFN-Bologna MoEDAL groups, combined with cutting edge image analysis software. This enables low NTD thresholds to be maintained despite large beam related backgrounds. The NTD detectors will be calibrated using the heavy-ion (Fe) beam at NASA's Space Radiation Laboratory Laboratory (NSRL).
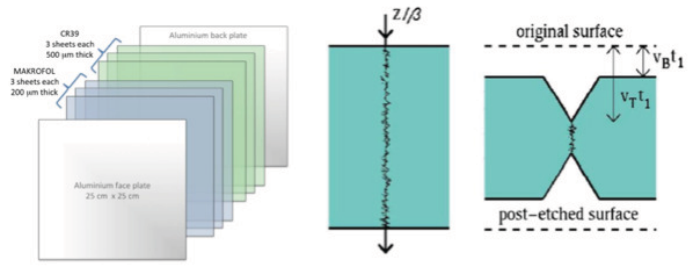

Figure 2. (Left) A MoEDAL stack comprised of CR39 and Markrofol NTD detectors. (Right) The damage zone created by the HIP that is subsequently revealed by etching in a hot $\mathrm{NaOH}$ solution. 
The Magnetic Monopole Trapper (MMT) is the third MoEDAL sub-detector system. Its sensitive volume consists of roughly 1 tonne of pure aluminium trapping volumes deployed around the MoEDAL cavern at IP8. Aluminium is well suited as it has an anomalously large nuclear magnetic moment. After exposure the MMT trapping volumes will be monitored at the ETH SQUID facility for the presence captured monopoles. A schematic description of this facility is shown in figure 3 (left). Figure 3 (right) shows the results of an exposure of a MMT test detector at IP8 on the LHC ring. As can be seen the SQUID can detect magnetic charges greater than one tenth of a Dirac charge. Test solenoids can be used to calibrate the response of the SQUID to a trapped monopole. After the SQUID scan has been performed the trapping volumes will be sent to an underground laboratory, for example SNOLAB, to be monitored for the decays of very long lived electrically charged particles.

The use of SQUIDS to detect trapped magnetic charge has also been thoroughly tested in particle and astroparticle experiments where the search has been performed on "found" or alternate use (such as beampipes) objects on a "one-off" basis. But, MoEDAL is the first experiment ever to use purposemade replaceable trapping volumes to capture magnetic charge. After the SQUID scan has been performed the trapping volumes will be sent to SNOLAB $-2 \mathrm{~km}$ underground - to be monitored for the decays of very long lived electrically charged particles.
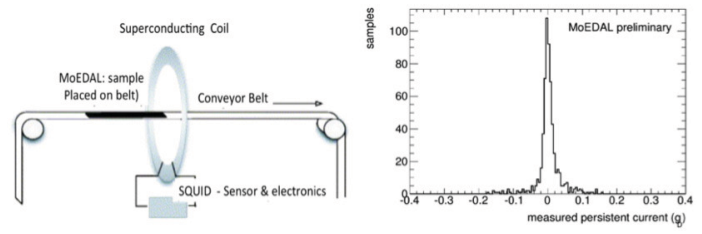

Figure 3. (Left) A schematic depiction of the use of a SQUID magnetometer to detect the presence of a trapped magnetic charge. (Right) The results of a SQUID scan of MMT elements exposed to roughly a year of LHC collisions at IP8 - as part of a MoEDAL test MMT detector deployment.

The fourth and only active sub-detector system is the TimePix pixel device array (TMPX), consisting of $\sim 8$ devices distributed throughout the MoEDAL cavern at IP8. TMPX will be used to monitor highly ionizing beam related backgrounds. Each pixel of the innovative TimePix chip contains a preamp, a discriminator with threshold adjustment, synchronization logic and a 14-bit counter. MoEDAL uses TimePix Time-over-Threshold modes where each pixel acts as an ADC for energy measurement. Effectively, the TimePix device is a tiny electronic bubble-chamber providing a realtime 'colour' (energy dependent) movie of the background as illustrated in figure 4.

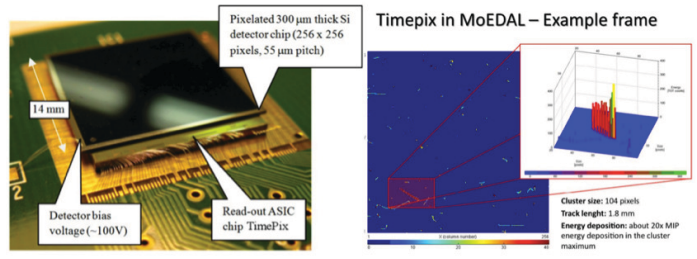

Figure 4. (Left) a photograph of a TimePix pixel device. (Right) A view of a low energy highly ionizing event in the TimePix chip's silicon sensitive volume. 


\subsection{A proposed minimally charged (millicharged) particle detector for MoEDAL}

A key aspect of the MoEDAL experiment is that is sensitive to HIP avatars of new physics for which the standard general-purpose LHC detectors are not optimized. MoEDAL is preparing a proposal to add a new subdetector that is sensitive to mQPs. This addition is consistent with the MoEDAL collaboration's aim to extend the physics reach of the LHC in a way that is complementary to the existing capability provided by the main LHC detectors since they have no sensitivity to mQPs with charge below $\sim 0.1 \mathrm{e}$.

The proposed detector is similar in principle to that employed on an earlier beam dump search for MMIPs at SLAC [10]. It will employ three deep liquid scintillator layers in triple coincidence with an acceptance of 0.01

\section{A Comparison of the MoEDAL detector with conventional LHC detectors}

MoEDAL's exposed NTD detectors are sent for analysis to INFN-Bologna and CERN and for calibration at the NASA Space Radiation Laboratory (NSRL) heavy-ion beam facility. The exposed MMT trapping detectors are monitored for the presence of trapped magnetic charge at the ETH Zurich SQUID Facility and at SNOLAB in Canada for captured long-lived massive electrically charged particles. In this way MoEDAL's unparalleled use of passive tracking and trapping detectors has the extraordinary effect of allowing the physics to be brought to the detectors, rather than the usual situation of bringing the detector to the physics. This unique approach has the effect of adding the capabilities of the remote facilities to that of the deployed MoEDAL detector and allowing us to significantly expand the physics discovery reach of the LHC in a cost effective way.

Standard collider detectors are not optimized for the detection of HIPs such as magnetic monopoles and massive long-lived or stable charged particles. Rather, the general-purpose detectors, such as ATLAS and CMS, are designed to detect minimum-ionizing particles moving near to the speed of light. Effects arising from the particles low velocity and high-density energy deposition, such as electronics saturation, light quenching in scintillators, and adjacent hits from delta electrons, are extremely challenging to deal with. Indeed, in some cases it may be impossible to make an accurate measurement of the effective charge of the particle. For example, the resulting dead time as a result of electronics saturation may be of the order of the bunch crossing time. In addition, HIPS will be quickly absorbed within the detector mass even before they penetrate far into the inner tracking detectors.
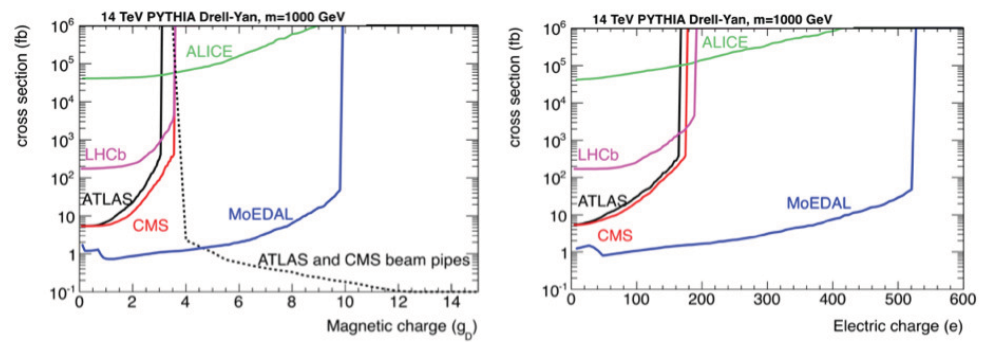

Figure 5. (The expected reach [11] of the search for direct monopole-anti-monopole Drell-Yan pair production process at the LHC $(\mathrm{Ecm}=14 \mathrm{TeV})$. Assuming the luminosity taken by LHCb/MoEDAL is $2 \mathrm{fb}^{-1}$, by ATLAS \& CMS $20 \mathrm{fb}^{-1}$, and by ALICE $0.004 \mathrm{fb}^{-1}$. 
On the other hand, MoEDAL does not suffer from the problems that would severely hamper detection of slow moving HIPS at the main LHC detectors. The NTD based sub-detectors have the demonstrated ability to measure accurately the ionizing power of a HIP. The MMT sub-detector, read out through a remote SQUID, can directly detect magnetic charge - no other Collider detector has this capability. MoEDAL's largely passive detection systems require no trigger, electronics, high voltage or gas supply. Its light and uniform detection technology requires minimal support structure and provides maximum cost effective coverage close to IP8 with few experimental compromises.

MoEDAL's sensitivity to highly ionizing messengers of new physics, together with its ability to make a permanent 'photographic' record of HIP BSM particles in its NTD detectors and capture such particles in its trapping detectors, is unique. This set of capabilities combined with the absence of SM backgrounds allows the definitive identification of HIP avatars of new physics with only a few candidate events detected. In an independent study [11] a comparison was made of the response of the LHC detectors to HIPs, as shown in figure 5. MoEDAL's sensitivity clearly surpasses that of the other LHC detectors.

\section{MoEDAL's Physics Program, a New Light on the Terascale Frontier}

MoEDAL's pioneering physics prospectus [9] covers more than 34 fundamentally important BSM scenarios allowing MoEDAL to significantly expand the LHC's discovery horizon in a complementary way. This program is illustrated in the pie chart shown in figure 6. One of the main objectives for MoEDAL is the state-of-the-art search for the magnetic charge, just as the search for the Higgs was the prime motivation for the LHC and its general-purpose experiments. For example, the work of our Theory Board has renewed interest in the LHC search for a Terascale EW Monopole [12] arising from the SM. If discovered, this would be the first topological particle to be observed - with the utmost consequences for our understanding of the Universe. But MoEDAL is designed to do much more as indicated in figure 6 and as discussed below.

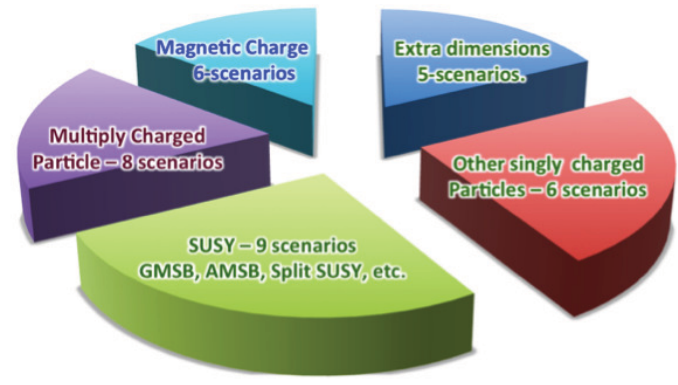

Figure 6. The MoEDAL Physics Program broken down by topic.

\subsection{The hunt for magnetic charge}

It was Dirac who first hypothesized that a magnetic monopole could exist within the framework of quantum mechanics [13]. He saw the monopole as the end of an infinitely thin infinitely long solenoid called a Dirac string. The annoying string does not pose a problem as long as it cannot be detected. Dirac surmised that the Dirac string would not detectable if the phase picked up by the wave function 
of the electron as it travels completely around the string is equal to one - in other words it has no affect. He calculated that the condition for the string not to be detected:

$$
e^{i q g}=1 \Rightarrow 2 \pi n(n=1,2,3 . . n)
$$

Dirac was quick to realize that the above relation between electric charge (q) and magnetic charge (g) explains the quantization of electric charge, as long as at least one monopole exists. The numerical value of the magnetic charge is $g=n / 2 \alpha=n 68.5 q$ - using $1 / \alpha=4 \pi / q^{2}$ where $\alpha$ is the fine structure constant $(\sim 1 / 137)$ giving rise to the extremely highly ionizing nature of the magnetic monopole that we discussed above.

Julian Schwinger [14] generalized the quantization condition to dyons, particles that carry both electric and magnetic charge. If we consider two dyons with electric and magnetic charges $\left(q_{1}, g_{1}\right)$ and (q2, g2) Schwinger found that each dyon is unable to detect the Dirac string of the other iff:

$$
q_{1} g_{2}-q_{2} g_{1}=n
$$

He found that the minimum charge for a megntic monopole was twice the Direc charge.

\subsection{The't Hooft Polyakov monopole}

In the Standard Model electroweak unification was achieved using a gauge theory developed by Sheldon Glashow, Steven Weinberg and Abdus Salam (GWS) [15]. This theory had two components called U(1) - that has the same Abelian gauge symmetry structure as Maxwell's electromagnetism and SU(2), that has a non-Abelian gauge symmetry that describes the weak interaction. These symmetries are broken at low energies by the Higgs field. In 1974 Gerard 't Hooft [16] and Alexander Polyakov [17] wrote their famous papers on a new kind of magnetic monopole that didn't have a problematic "Dirac String" with a point-like singularity at the origin. Their monopole had its origin in Georgi's and Glashow's earlier model of EW unification [18] later supplanted by GWS theory.

The Georgi-Glashow model has only one gauge symmetry, a non-Abelian symmetry known as $\mathrm{SO}(3)$, that is spontaneously broken by the Higgs field down to the U(1) gauge symmetry of Maxwell's equations. The quantization of charge arises naturally in such theories without the need for a magnetic monopole as was the case in Dirac's theory. However, a major implication of 't Hooft's and Polyakov's work was that any unified gauge theory like Georgi's and Glashow's inevitably possesses monopole solutions. So, magnetic monopoles that were only "nice" in Abelian theories like Maxwell's electromagnetism are now necessary in some non-Abelian theories.

The 't Hooft and Polyakov monopole arose in the situation where the Higgs field vector points away from the origin everywhere in the "hedgehog" configuration, In contrast to the Dirac monopole, which necessitates a singular point-like magnetic source at the origin, the 't Hooft-Polyakov monopole is everywhere smooth. In this case the field configuration is topologically stable, like a knot that cannot unravel. Remarkably, the Higgs-field configuration corresponding to this magnetic monopole is an example of a topological soliton, a localized and stable field excitation that behaves like an elementary particle. In the microscopic heart of the 't Hooft-Polyakov monopole the full unbroken $\mathrm{SO}(3)$ gauge symmetry of the unified theory reigns supreme. Far away the 't Hooft-Polyakov monopole would be indistinguishable from a Dirac monopole except that its magnetic charge is twice as much.

The roughly $100 \mathrm{GeV} / \mathrm{c}^{2}$ mass of these monopoles - determined by the energy scale of the weak interaction - was ruled out by existing experimental results. But 't Hooft and Polyakov observed that the structure of the SU(5) Grand Unified Theory (GUT), which attempts to describe all known elementary particle forces except gravity, was similar in some respects to that of the Georgi-Glashow model. It was not then surprising that the SU(5) GUT model also contains a 't Hooft-Polyakov magnetic 
monopole solution, but with the much higher mass of $10^{16} \mathrm{GeV}$. The existence of magnetic monopoles is an unavoidable consequence of any theory that would describe all strong and electroweak forces by one GUT interaction. Unfortunately, GUT monopoles are far too massive be produced at any conceivable man-made accelerator.

\subsection{The electroweak monopole}

The discovery of what increasingly appears to be the Standard Model Higgs boson in July 2012 by the ATLAS and CMS [19] experiments at the Large Hadron Collider (LHC) at CERN has led to the possibility that the last piece of the Standard Model puzzle may now be in place. This discovery reinforces the electroweak theory of Glashow, Salam and Weinberg, a central pillar of the successful Standard Model. A monopole-like solution within the framework of the Standard Model would explain from first principles the quantization of the electric charge, something that the present Standard Model cannot do. In 1986 Cho and Maison published a paper suggesting that such a magnetic monopole could exist.

Cho and Maison's "Electroweak" monopole is a hybrid of the Dirac Monopole and the 't Hooft Polyakov monopole. Like the 't Hooft-Polyakov monopole its magnetic charge is twice that of the Dirac monopole. According to a recent estimate [9] the mass of the Electroweak Monopole could lie in the range 4-7 GeV/c ${ }^{2}$, possibly low enough to be detectable at the LHC by the MoEDAL experiment. Thus, the LHC could be the first collider to produce Electroweak monopoles. Naively, one might expect the production rate would be comparable to a typical electroweak process such as WW production, above the threshold energy. If that is so then MoEDAL experiment should easily be able to detect the EW monopole, if it the threshold is within reach at the LHC. If detected the Electroweak monopole would be the first topological particle to be observed in nature.

\section{Electrically charged highly ionizing partilce messengers of new physics}

Electrically charged stable or pseudo-stable massive particles (SMPs), with mass greater than the proton mass do not exist within the framework of the Standard Model - they are true and certain messengers of new physics, predicted in a plethora of beyond the Standard Model scenarios. As we have seen such charged massive particles can be highly ionizing, if they have a combination of low velocity and/or multiple charge, and are thus detectable by MoEDAL. The most obvious possibility for an SMP is that one or more new states exist which carry a new conserved, or almost conserved, global quantum number. SUSY with R-parity, extra dimensions with KK-parity, and several other models fall into this category. The lightest of the new states will be stable, due to the conservation of this new 'parity', and depending on quantum numbers, mass spectra, and interaction strengths, one or more higher-lying states may also be stable or meta-stable.

In general, electrically charged stable states are on the face of it highly constrained by cosmology. Coloured particles are also strongly constrained. Consequently, most models are usually constructed to provide non-charged and colourless stable dark-matter candidates. For those models where Weakly Interacting Massive Particles (WIMPs) are the dark matter candidate, SMPs must be meta-stable states that are higher in the hierarchy of particles. Models with long-lived or stable SMPs either have a nonWIMP dark matter candidate or else do not address the problem of particulate dark matter at all. Our recent paper [9] describing MoEDAL's physics program describes nearly thirty such new physics scenarios involving electrically charged massive particles that are "detector stable" and can have a $Z / \beta$ or at least around five. 


\subsection{Highly ionizing particles from supersymmetric scenarios}

One key problem of the Standard Model is that it does not include gravity, one of the four fundamental forces. The model also fails to explain why gravity is so much weaker than the electromagnetic or nuclear forces. For example, an electrostatically charged hair comb can counteract the gravitational attraction of a whole planet on a small scrap of paper. This difference in the strength of fundamental forces is one aspect of the "Hierarchy Problem"'.

A prime candidate for a deeper more fundamental theory that would resolve many of the important problems with the Standard Model is Supersymmetry (SUSY), where for every Standard Model particle there is a superpartner (sparticle) whose spin differs by $1 / 2$ - for excellent reviews see, e.g. [20]. By doubling the number of particles in this way one obtains cancellations and degrees of freedom with which we can tackle such the Hierarchy Problem. Indeed, SUSY appears to be a necessary ingredient in the only available candidate for quantum theory of gravity, string theory.

If SUSY were an exact symmetry, SUSY particles would have the same masses as their SM partners. But as no SUSY particles have been observed to date we can surmise that the SUSY partners must be much heavier than the Standard Model particles and therefore SUSY must be a broken symmetry. The mass gap between Standard Model and SUSY particles cannot be much larger than $\sim 1$ TeV without destroying SUSY's ability to solve the hierarchy problem. Other important benefits of SUSY are that it allows for the unification of the electromagnetic, weak and strong gauge couplings of the Standard Model - a necessary and predicts particles that are natural dark matter candidates. The Standard Model fails in both these arenas.

At present, little is known for certain about the nature of the SUSY-breaking mechanism. As a result the MoEDAL physics program explores many supersymmetric scenarios [9]. The most common SUSY framework is divided into two sectors. The usual fields of the Minimal Supersymmetric extension to the Standard Model (MSSM) i.e. quarks, leptons, gauge bosons, and two Higgs doublets, together with their supersymmetric partners, belong to the visible sector. While the second, hidden, sector contains additional fields - yet unobserved quantum superfields and the corresponding hypothetical particles, which do not interact via the Standard Model force messengers (gluons, photons, and $\mathrm{W}$ and $\mathrm{Z}$ bosons) - that lead to the breaking of Supersymmetry. The SUSY breaking, occurring at some higher energy scale in the hidden sector, is mediated to the visible sector by the exchange of weakly interacting 'messenger' particles.

As an example we consider the search for long lived stau slepton, the superpartner of the tau lepton, just one of many examples of SUSY scenarios that give rise to SMPs detectable by MoEDAL [9]. At the LHC, the signals of the supersymmetric particles depend on the nature of the lightest SUSY particle. It may be the lightest neutralino, which escapes detection and leads to missing transverse energy in the detector. Another possibility for the LSP is the gravitino $(\tilde{G})$, the superpartner of the graviton. If the LSP is the gravitino the lifetime of the NLSP slepton, decaying via $\tilde{l} \rightarrow \tilde{G} l$, is given by [21]:

$$
T=\frac{1}{\Gamma(\tilde{l} \rightarrow \tilde{G} l)}=\frac{1}{48 \pi M_{P l}} \frac{m_{l}^{5}}{M_{\tilde{G}}^{2}}\left[1-\frac{M_{\tilde{G}}^{2}}{m_{l}^{2}}\right]^{4}
$$

where, $M_{P l}$ is the Planck Scale, $M_{\tilde{G}}$ is the mass of the gravitino, $m_{\tilde{l}}$ is the mass of the slepton for example a stau, the superpartner of the tau. Since $M_{P l}$ is much larger than the electroweak scale, the NLSP lifetime is naturally long, perhaps reaching into years, as can be seen in figure 7.

MoEDAL can detect massive slowly moving $(\beta<0.2)$ very long-lived sleptons in two ways. First, via the high ionization which will leave a signal in MoEDAL's NTD detectors. Secondly, a fraction of the sleptons produced will be captured in its trapping detectors. After exposure at IP8 the 


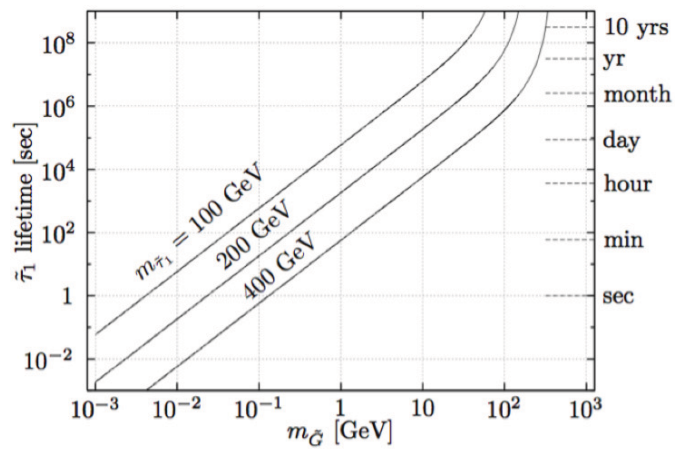

Figure 7. (The stau lifetime in a gravitino LSP scenario, for different values of the stau $(\tilde{\tau})$ mass and the gravitino $(\tilde{G})$ mass [21].

trapping volumes will be removed and monitored deep underground, where cosmic ray backgrounds are minimised, for the slow decays of any trapped sleptons. MoEDAL's sensitivity to such events complements that of the standard general-purpose LHC detectors ATLAS and CMS that are optimized for minimum ionizing relativistic particles. On the other hand, MoEDAL is optimized for HIPs.

\section{Millicharged matter}

Although Charged Massive Particles (ChaMPs) have been more or less ruled out as dark matter candidates, this is not easily the case for particles with tiny charge. Millicharged particles (mQPs) have been firstly proposed as a solution of the dark matter puzzle long time ago [22]. It is possible to simply add mQPs to the Standard Model for example as Standard Model neutrinos that have tiny electric charges. A small modification of the hypercharge assignments of the Standard Model particles can accommodate tiny electric charges to neutrinos (for a review see e.g. [23]).

Holdom [24] has shown that mQPs will arise naturally, and without violating charge quantization, in certain models involving what are called "shadow universes" or "mirror universes" that are hidden from our own. If there is a shadow-U(1) gauge group i.e a shadow electromagnetism, it follows there is such a thing as a shadow photon, also called a paraphoton. Holdom showed that if the photon and the paraphoton mix a particle charged under the Shadow-U(1) appears to have a very small coupling to the photon - it is a mQP.

The $\mathrm{mQP}$ parameter space as defined by mass and charge is highly constrained by direct searches by accelerator experiments [26][27][28][29] as well as indirect astrophysical [26],[30], [31],[32] and cosmological observations [25][30][33]. Of course, indirect observations can be evaded by adding extra degrees of freedom or by using different assumptions. In addition, the parameter space for mQPs with masses between $0.1 \mathrm{GeV}$ and $100 \mathrm{GeV}$ is largely unexplored by accelerator based experiments. MoEDAL's proposed mQP subdetector should be able to push into uncovered territory towards the discovery limits shown in figure 8 for $\mathrm{mQPs}$ with charge $\geq 0.001 \mathrm{e}$.

\section{Cosmic MoEDAL}

Grand Unified Theories (GUTs) of electroweak and strong interactions predict the existence of superheavy magnetic monopoles with masses larger than $10^{16} \mathrm{GeV}$ [35]. They would have been produced at the end of the GUT epoch, at a mass scale of $\sim 10^{14} \mathrm{GeV}$ and the cosmic time of $\sim 10^{-} 34$ 


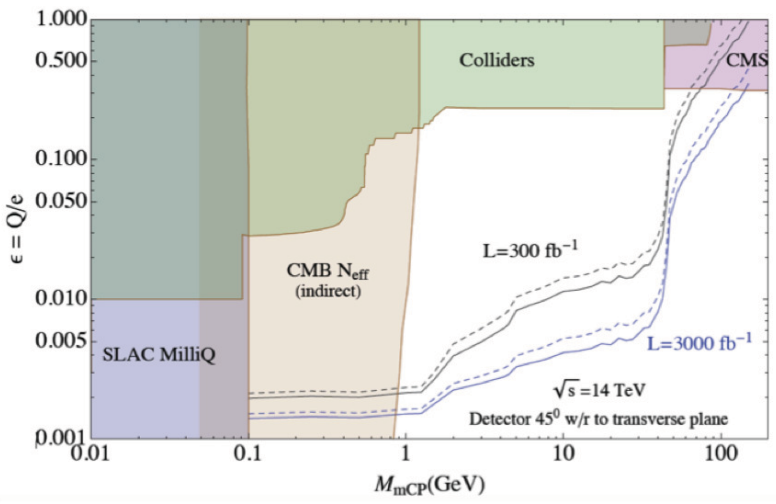

Figure 8. The existing bounds are taken from Ref. [25]. The black line shows the expected 95\% C.L. exclusion (solid) and $3 \sigma$ sensitivity (dashed) of the proposed experiment at centre-of-mass energy $14 \mathrm{TeV}$, assuming 300 $\mathrm{fb}^{-1}$ of luminosity. In blue the corresponding limits achievable in an LHC run with $3000 \mathrm{fb}^{-1}$

s. Such monopoles cannot be produced with existing accelerators, nor with any foreseen for the future. Lower mass monopoles, proposed by many authors, require a phase transition in the early universe in which a semi-simple gauge group yields a U(1) factor at a lower energy scale [35][36]. Such"Intermediate Mass Monopole" (IMMs), with masses around $10^{6} \rightarrow 10^{10} \mathrm{GeV}$, are discussed elsewhere [36][37][38][39]. Additionally, monopoles with masses in the range that are potentially detectable at the LHC have been posited [9]. Such monopoles would be present as primary cosmic particles but also as secondaries produced in UHECR interactions in the atmosphere.

For example, an exposure at a high altitude laboratory would allow us to push the search for relativistic and non-relativistic cosmic monopoles down to relatively low masses (compared to the GUT monopole), higher magnetic charges and lower velocities [40]. The high altitude exposure could allow detection of the above mentioned particles even if they had a strong interaction cross section as may be the case of the monopole-proton dyonic composite and the lower mass nuclearite - which could prevent them from reaching the earth surface

A large array of NTDs, of the order of $10,000 \mathrm{~m}^{2}$ or larger, deployed for several years at high altitude would be able to push the search for HIPs in primary or secondary cosmic rays beyond the level reached by previous experiments such as SLIM high altitude array and the MACRO experiment underground. In particular, such a surface array would be sensitive to relativistic and non-relativistic magnetic monopoles with multiple magnetic charge, fluxes well under the Parker Bound, velocities down to $\beta \sim 10^{-4}$ and masses ranging from the TeV level up to the highest GUT monopole mass. The high altitude would allow for an increased possibility that particles which may have a greater than electromagnetic cross-section - as may be the case for a lower mass nuclearite or a dyonic monopoleproton bound state - can be detected. In this arena it would seem most advantageous to place an array at the highest possible altitude, for example on: Mt Chacaltaya in Bolivia ( $5300 \mathrm{~m})$; Tenerife on the Canarie Islands ( 4000m); or, Mauna Kea in Hawaii (4200 m).

\section{Summary and Conclusion}

The MoEDAL detector employing unconventional methodologies and tuned to the prospect of discovery physics was installed at Point 8 on the LHC rings in the Winter of 2014/2015. The largely 
passive MoEDAL detector, has a dual nature. First, it acts like a giant camera, comprised of nuclear track detectors - analyzed offline by ultra fast scanning microscopes - sensitive only to new physics. Second, it is uniquely able to trap the particle messengers of physics beyond the Standard Model for further study. MoEDAL's radiation environment is monitored by a state-of-the-art real-time TimePix pixel detector array. The possibility of adding a new MoEDAL subdetector sensitive to mQPs is being studied.

In 2015 the LHC restarted operations at the unparalleled energy of nearly $6.5 \mathrm{TeV}$ beam energy and the enormous collision rate of around a billion collisions per second. The LHC has been compared to an immense microscope allowing us to peer deeper into the Terascale quantum universe than ever before, to explore the inner structure of matter at a scale down to $10^{-19} \mathrm{~m}$. The LHC will recreate the conditions that occurred when the Universe was a picosecond old providing an unprecedented laboratory for the study of the cosmology of the nascent Universe at the earliest times. In parallel, non-accelerator experiments are also exploring the Terauniverse via high-energy astrophysics.

At present it looks very much as though the Higgs boson - the last piece of the Standard Model has been discovered. But, the LHC is only just getting started. The quest for the deeper underlying theory has now begun in earnest that brings many fundamental questions to the fore. Are there new symmetries of nature? Are there extra spatial dimensions? Is there a deeper substructure? Does magnetic charge exist? Why is gravity so weak compared to the other fundamental forces? What is the nature of dark matter? What was the physics of the earliest era of the universe? The list goes on.

The MoEDAL experiment has a huge discovery potential designed to expand the physics horizons of the LHC and provide paradigm-shifting insights into foundational questions in a complementary way to the other LHC experiments due to its enhanced sensitivity to the highly ionizing avatars of new physics such as magnetic monopoles or massive (pseudo-)stable charged particles. We are investigating the possibility to further enhance the complementary extension of the LHC's physics reach by incorporating a new sub-detector to search for minimally ionizing particles. The unprecedented but complementary nature of the MoEDAL detector and its systematics - exemplified by its ability to retain a permanent record, and even capture new particles for further study - will make MoEDAL an invaluable asset in the elucidation of any Terascale Beyond the Standard Model (BSM) scenario covered by its extensive physics repertoire.

The search for HIPs such as Q-balls, nuclearites and magnetic monopoles can be pushed to GUT mass scales, and below the Parker Bound [41], by the deployment of a 10,000 $\mathrm{m}^{2}$ or larger COSMICMoEDAL array at high altitude.

\section{References}

[1] J. L. Pinfold, MoEDAL Collaboration, Technical Design Report of the MoEDAL Experiment MoEDAL Collaboration, CERN-LHCC-2009-006, MoEDAL-TDR-001 (2009).

[2] Large Hadron Collider Official Site: http://home.web.cern.ch/topics/large-hadron-collider.

[3] G. Aad, et al., [ATLAS Collaboration], JINST 3, S08003 (2008).

[4] S. Chatrchyan et al., [CMS Collaboration], JINST 3, S08004 (2008).

[5] K. Aamodt et al., [ALICE Collaboration], JINST 3, S08002 (2008).

[6] A. A. Alves, Jr. et al., [LHCb Collaboration], JINST 3, S08005 (2008).

[7] G. Anelli et al., [TOTEM Collaboration], JINST 3, S08007 (2008).

[8] O. Adriani et al., [LHCf Collaboration] JINST 3, S08006 (2008).

[9] B. Acharya et al., [MoEDAL Collaboration], Int. J. Mod. Phys. A29, 1430050 (2014).

[10] A.A. Prinz, R. Baggs, J. Ballam, S. Ecklund, C. Fertig, J.A. Jaros, K. Kase, A. Kulikov, Willem

G. J. Langeveld, R. Leonard et al., Phys. Rev. Lett. 811175 (1998). 
[11] A. de Roeck et al., Eur. Phys. J. C72, 1985 (2012)

[12] K. Kimm, J. H. Yoon and Y. M. Cho, Eur. Phys., J. C75 67 (2015).

[13] P. A. M. Dirac, Proc. Roy. Soc. Lond. A133, 60 (1931)

[14] J. S. Schwinger, Science 165757 (1969).

[15] S. L. Glashow, Nucl. Phys. 22 no. 4579 (1961); S. Weinberg, Phys. Rev. Lett. 19, 1264 (1967);

A. Salam, Weak and electromagnetic interactions, in Elementary particle physics: relativistic groups and analyticity, N. Svartholm, ed., p. 367. Almqvist \& Wiskell, Proceedings of the eighth Nobel symposium (1968); G. 't Hooft and M. Veltman, Nucl. Phys. B44, 189 (1972).

[16] G. 't Hooft, Nucl. Phys. B79, 276 (1974).

[17] A. M. Polyakov, JETP Lett. 20, 194 (1974).

[18] H. Georgi and S. Glashow, Phys. Rev. Lett. 32, 438 (1974).

[19] G. Aad et al., ATLAS Collaboration, Phys. Lett. B716, 1 (2012); S. Chatrchya et al., CMS Collaboration Phys. Lett. B716, 30 (2012).

[20] ] S.P. Martin, hep-ph/9709356 (1997); X. Tata, hep-ph/9706307 (1997).

[21] K. Hamaguchi, M. M. Nojiri and A. de Roeck, JHEP 0703, 046 (2007).

[22] H. Goldberg and L. J. Hall, Phys. Lett. B174, 151 (1986); A. De Rujula, S. L. Glashow and U. Sarid, Nucl. Phys. B333, 173 (1990); S. Dimopoulos, D. Eichler, R. Esmailzadeh and G. D. Starkman, Phys. Rev. D41, 2388 (1990).

[23] R. Foot, G. C. Joshi, H. Lew and R. R. Volkas, Mod. Phys. Lett. A5, 95 (1990) [Erratum-ibid. 1990 A 5, 2085]; R. Foot, H. Lew and R. R. Volkas, J. Phys. G19, 361 (1993) [Erratum-ibid. 1993

G 19, 1067]; S. Davidson, S. Hannestad and G. Raffelt, JHEP 0005, 003 (2000).

[24] B. Holdom, Phys. Lett. B166, 196 (1986).

[25] H. Vogel and J. Redondo, JCAP 1402, 029 (2014).

[26] S. Davidson, S. Hannestad, and G. Raffelt, JHEP 0005, 003 (2000).

[27] A. Prinz, R. Baggs, J. Ballam, S. Ecklund, C. Fertig, et al., Phys. Rev. Lett. 811175 (1998).

[28] A. Badertscher et al., Phys.Rev. D75, 032004 (2007).

[29] S. Chatrchyan et al. (CMS Collaboration), Phys. Rev. D87 092008 (2013).

[30] S. Davidson, B. Campbell, and D. C. Bailey, Phys. Rev. D43, 2314 (1991).

[31] R. Mohapatra and I. Rothstein, Phys. Lett. B247, 593 (1990).

[32] S. Davidson and M. E. Peskin, Phys. Rev. D49, 2114 (1994).

[33] A. Dolgov, S. Dubovsky, G. Rubtsov, and I. Tkachev, Phys. Rev. D88, 117701 (2013).

[34] A. Haas et al., [hep-ph] arXiv:1410.6816v1 (2014).

[35] Monopoles in Quantum Field Theory, N.S. Craige, P. Goddard, W. Nahm editors, World Scientific (1982); G. Giacomelli, Rivista del Nuovo Cimento 7 (1984); Monopoles in Gauge Theories, Lecture Notes by N. Craige, G. Giacomelli, W. Nahm and Q. Shafi, World Scientific (1986).

[36] E. Huguet and P. Peter (Bound states in monopoles: sources for UHECR?) hep-ph/9901370 (1999).

[37] T. J. Weiler and T. K. Kephart, Nucl. Phys. B. Proc. Suppl. 51, 218 (1996); T.W. Kephart and T.

J. Weiler, Astrop. Phys. 4, 217 (1996).

[38] C. O. Escobar and R. A. Vazquez, Astrop. Phys. 10, 197 (1999).

[39] A. De Rujula, Nucl. Phys. B435, 257 (1995).

[40] ] S. Balestra et al., Eur.Phys.J. C55, 57-63 (2008).

[41] E. Parker, Astrophys. J. 160, 383 (1970). 